University of Nebraska - Lincoln

DigitalCommons@University of Nebraska - Lincoln

July 1998

\title{
Elementary excitation spectrum of a trapped weakly interacting Bose-Einstein condensate
}

\author{
Mircea Marinescu \\ University of Nebraska - Lincoln \\ Anthony F. Starace \\ University of Nebraska-Lincoln, astarace1@unl.edu
}

Follow this and additional works at: https://digitalcommons.unl.edu/physicsstarace

Part of the Physics Commons

Marinescu, Mircea and Starace, Anthony F., "Elementary excitation spectrum of a trapped weakly interacting Bose-Einstein condensate" (1998). Anthony F. Starace Publications. 60.

https://digitalcommons.unl.edu/physicsstarace/60

This Article is brought to you for free and open access by the Research Papers in Physics and Astronomy at DigitalCommons@University of Nebraska - Lincoln. It has been accepted for inclusion in Anthony F. Starace Publications by an authorized administrator of DigitalCommons@University of Nebraska - Lincoln. 


\title{
Elementary excitation spectrum of a trapped weakly interacting Bose-Einstein condensate
}

\author{
Mircea Marinescu and Anthony F. Starace \\ Department of Physics and Astronomy, The University of Nebraska, 116 Brace Laboratory, Lincoln, Nebraska 68588-0111
}

(Received 13 May 1996; revised manuscript received 6 February 1997)

\begin{abstract}
An analytic expression is presented for the elementary excitation spectrum of the Bose-Einstein condensate of a trapped boson system in the weakly interacting, low-density limit. Explicit analytic formulas for the elementary excitation spectrum are obtained for harmonic-oscillator traps. These formulas provide information about the behavior of the elementary excitation levels as a function of the number of atoms and their interaction strength for a given trap geometry. They also provide a low-density benchmark for results of fully numerical calculations. [S1050-2947(97)01207-9]
\end{abstract}

PACS number(s): 03.75.Fi, 05.30.Jp, 67.90.+z

\section{INTRODUCTION}

Recent experimental successes in achieving Bose-Einstein condensation (BEC) in various systems of trapped and cooled alkali-metal atoms [1-3] have stimulated a growing interest in theoretical study of BEC [4-9] in an external potential. Still more recently, there have been both experimental [9] and theoretical [10-12] studies of the excitation spectrum of the condensate. In this paper we consider a weakly interacting condensate in a trap and obtain a perturbative analytic expression for its elementary excitation spectrum (EES). This analytic expression allows one to easily obtain information about the dependence of the EES on the number of atoms in the trap and on the particular trap geometry. Furthermore, just as the analytic result of Stringari [10] provides a benchmark for fully numerical calculations $[11,12]$ in the limit of high trap densities, our formula provides a similar benchmark in the limit of low trap densities. As applications, we provide explicit formulas for the EES of isotropic, axially symmetric, and anisotropic harmonicoscillator potential traps. For the case of axially symmetric harmonic-oscillator traps we show that our analytic formula provides results that agree very well with the nonperturbative, numerical results of Edwards et al. [11] and of Esry [12] in the limit of low densities.

In Sec. II, we briefly summarize the well-known generalized canonical transformation for diagonalizing the Hamiltonian of the trapped boson condensate. In Sec. III we present our analytic expression for the EES for the case of a low-density condensate. In Sec. IV we present explicit analytic expressions for the energy-level spectra of isotropic, axially symmetric, and anisotropic harmonic-oscillator potential traps. Finally, in Sec. V we discuss our results and contrast them with Stringari's [10] analytic result for the high-density limit. We also make comparisons with nonperturbative, numerical results for the low-density limit $[11,12]$. Throughout this paper we use atomic units $\left(\hbar=m_{e}=e=1\right)$.

\section{GENERALIZED CANONICAL TRANSFORMATION}

Our analysis is based on a well-known [13-15] generalization of the Bogolubov [16] canonical transformation in order to bring the following type of Hamiltonian to a diagonal form:

$$
H=\left[a^{\dagger}\right]^{T} A[a]+[a]^{T} B[a]+\left[a^{\dagger}\right]^{T} B^{*}\left[a^{\dagger}\right],
$$

where $A$ is a Hermitian matrix, $B$ is a complex symmetric one, and $[a]$ and $\left[a^{\dagger}\right]$ are the column matrices of boson annihilation and creation operators, respectively. We use the superscript $T$ to denote the transpose operator; $[a]^{T}$ and $\left[a^{\dagger}\right]^{T}$ are row matrices.

The generalized canonical transformation (GCT) of the annihilation and creation operators is

$$
\begin{aligned}
& {[a]=\alpha[b]+\beta\left[b^{\dagger}\right],} \\
& {\left[a^{\dagger}\right]=\alpha\left[b^{\dagger}\right]+\beta[b],}
\end{aligned}
$$

where $b_{n}$ and $b_{n}^{\dagger}$ are two new sets of boson annihilation and creation operators (also called the quasiparticle operators) and $\alpha$ and $\beta$ are two real matrices that must obey the relations

$$
\begin{aligned}
& \alpha \alpha^{\dagger}-\beta \beta^{\dagger}=1, \\
& \alpha \beta^{\dagger}-\beta \alpha^{\dagger}=0,
\end{aligned}
$$

in order that $b_{n}$ and $b_{n}^{\dagger}$ satisfy the boson commutation relations. Equations (2.4) and (2.5) imply that $(\alpha+\beta)^{T}$ is the inverse matrix of $(\alpha-\beta)$,

$$
(\alpha-\beta)^{-1}=(\alpha+\beta)^{T} .
$$

The matrices $\alpha$ and $\beta$ are determined by requiring that the Hamiltonian in Eq. (2.1) is transformed to a diagonal form upon making the substitutions in Eqs. (2.2) and (2.3). One obtains, by straightforward matrix algebra [13-15],

$$
H=\frac{1}{2} \operatorname{Tr}[D(E)-A]+\left[b^{\dagger}\right]^{T} D(E)[b],
$$

where $D(E)$ is a diagonal matrix having the diagonal elements $E_{n}$, the $n$th quasienergy, which we shall later interpret to give the EES. The result from Eq. (2.7) is possible only if the following equations are satisfied:

$$
(\alpha \pm \beta)^{T}\left(A^{\mathrm{Re}} \pm 2 B^{\mathrm{Re}}\right)(\alpha \pm \beta)=D(E)
$$


where the superscript Re denotes the real part. The two relations in Eq. (2.8) are often called the equations for the EES. We mention that although the matrices $A^{\mathrm{Re}} \pm 2 B^{\mathrm{Re}}$ are real and symmetric, neither of Eqs. (2.8) is an eigenvalue equation since the $\alpha \pm \beta$ matrices are not unitary. However, multiplying Eq. (2.8) for + by Eq. (2.8) for - , one obtains

$$
(\alpha+\beta)^{T}\left[\left(A^{\mathrm{Re}}+2 B^{\mathrm{Re}}\right)\left(A^{\mathrm{Re}}-2 B^{\mathrm{Re}}\right)\right](\alpha-\beta)=D\left(E^{2}\right),
$$

which is a generalized eigenvalue problem since $\left(A^{\mathrm{Re}}+2 B^{\mathrm{Re}}\right)\left(A^{\mathrm{Re}}-2 B^{\mathrm{Re}}\right)$ is not a symmetric matrix. In Eq. (2.9) $D\left(E^{2}\right)$ denotes the square of the $D(E)$ diagonal matrix. One may interpret Eq. (2.9) as follows: $(\alpha-\beta)[(\alpha+\beta)]$ is a matrix whose columns are the right [left] eigenvectors of $\left(A^{\mathrm{Re}}+2 B^{\mathrm{Re}}\right)\left(A^{\mathrm{Re}}-2 B^{\mathrm{Re}}\right)$. Thus the generalized eigenvalue problem [Eq. (2.9)] completely defines the $\alpha$ and $\beta$ matrices and consequently the GCT [Eqs. (2.2) and (2.3)]. Equation (2.9) is the starting point of our calculation of the EES.

\section{ELEMENTARY EXCITATION SPECTRUM OF A TRAPPED BOSE-EINSTEIN CONDENSATE}

The effective Hamiltonian for $N$ interacting bosons is, in second quantization,

$$
\begin{aligned}
H= & \sum_{n} \epsilon_{n}^{0} a_{n}^{\dagger} a_{n} \\
& +\frac{1}{2} \sum_{n_{1}, n_{2}, n_{3}, n_{4}}\left\langle\phi_{n_{1}} \phi_{n_{2}}|V| \phi_{n_{3}} \phi_{n_{4}}\right\rangle a_{n_{1}}^{\dagger} a_{n_{2}}^{\dagger} a_{n_{4}} a_{n_{3}},
\end{aligned}
$$

where $a_{n}$ and $a_{n}^{\dagger}$ are the $n$th annihilation and creation operators for trap states and $\epsilon_{n}^{0}$ and $\phi_{n}$ are the $n$th eigenvalue and eigenfunction of the Schrödinger equation for bosons confined in the trap potential $U_{0}(\vec{r})$, i.e.,

$$
\left(\frac{P^{2}}{2 M}+U_{0}\right) \phi_{n}=\epsilon_{n}^{0} \phi_{n}
$$

In Eq. (3.2) $M$ is the mass of the boson and in Eq. (3.1) $V$ is the interaction potential between two bosons. Interaction terms involving three or more particles are neglected. Also, we assume that the range of the effective interaction between two bosons is much smaller than the size of the potential trap. Thus the matrix elements of $V$ in Eq. (3.1) may be evaluated in the long-wavelength approximation (i.e., ignoring the variation of the wave functions over the effective range of the interaction $V$ ) as

$$
\left\langle\phi_{n_{1}} \phi_{n_{2}}|V| \phi_{n_{3}} \phi_{n_{4}}\right\rangle \simeq g \int d \vec{r} \phi_{n_{1}}(\vec{r}) \phi_{n_{2}}(\vec{r}) \phi_{n_{3}}(\vec{r}) \phi_{n_{4}}(\vec{r}),
$$

where

$$
g=\int V(\vec{R}) d \vec{R}
$$

The parameter $g$ characterizes the effective interaction between two bosons; it has the dimension of (energy) $\times($ volume $)$.
The approximation in Eq. (3.3) is appropriate only if $g$ has a finite value, which depends on the effective potential being an integrable function. While it is not rigorously established what expression for the effective interaction between two atoms (bosons) in a trap should be introduced in Eq. (3.4), ${ }^{1}$ a widely used approximation for $g$ is $[5-7,10-12]$

$$
g=\frac{4 \pi a_{s c}}{M}
$$

where $a_{s c}$ is the scattering length. We adopt this approximation here.

The reduction of the $N$ interacting boson Hamiltonian in Eq. (3.1) to a quadratic, effective Hamiltonian proceeds in a way similar to that used to obtain the Bogolubov model Hamiltonian [16], but for a system of interacting bosons in a trap. The procedure may be described briefly as follows. Since the entire formalism is at $T=0$, one may assume that most of the atoms are in the ground state of the trap. Thus the operators $a_{0}$ and $a_{0}^{\dagger}$ may be replaced by the $c$ number $\sqrt{N}_{0}$, where $N_{0}$ is the number of atoms in the ground state of the trap. From the resulting expression, only terms proportional to $N_{0}$ and $N_{0}^{2}$ are retained. This approximation is compatible with the assumption that $N_{0}$ is large, i.e., $N_{0} \gg \sqrt{N}_{0}$, and that almost all atoms are in the ground state of the trap, $N-N_{0} \ll N[16,20]$, where $N$ is the total number of atoms. For this latter reason, terms of order $N_{0}^{3 / 2}$, which involve single excitations out the ground state, are ignored (as done, e.g., in Ref. [14]). $N_{0}$ is then replaced by the expression $N-\Sigma_{n}^{\prime} a_{n}^{\dagger} a_{n}$, where the prime to the summation indicates that the term $n=0$ is omitted. The result is

$$
H=W_{0}+\left[a^{\dagger}\right]^{T} A[a]+[a]^{T} B[a]+\left[a^{\dagger}\right]^{T} B\left[a^{\dagger}\right],
$$

where $W_{0}$, given by

$$
W_{0}=\varepsilon_{0} N+\frac{N^{2}}{2} g h_{00},
$$

is a $c$ number since $N$ and $g$ are assumed to be parameters and $A$ and $B$ are two real matrices whose elements are

$$
A_{n m}=\left(\varepsilon_{n}-\varepsilon_{0}-N g h_{00}\right) \delta_{n m}+2 N g h_{n m},
$$

\footnotetext{
${ }^{1}$ A difficulty in defining $g$ arises from the strongly repulsive behavior of $V(R)$ for small $R$ owing to the Pauli exclusion principle for the fermion particle constituents of the Bose atom composite particles [17]. It has been suggested [4] that the widely used Fermi $\delta$ potential [18] plus a short-range attractive potential may be both realistic and suitable for use in Eq. (3.4). Actually, a possible solution to this question may be given by the classical approach of van Kampen [19]. There the diatomic potential is split into a (negative) attractive part and a (positive) repulsive wall, which in turn may be treated as a $\delta$ potential (as in the case of a hard-sphere potential [18]). In this way the structure of the bound states that characterize the diatomic alkali-metal potential is included in the formalism. On the other hand, it is not clear what the correction to the scattering length stemming from the trap potential is.
} 


$$
B_{n m}=\frac{N}{2} g h_{n m}
$$

the $h_{n m}$ in Eqs. (3.7)-(3.9) are given by the integrals

$$
h_{n m}=\int \phi_{0}^{2}(\vec{r}) \phi_{n}(\vec{r}) \phi_{m}(\vec{r}) d \vec{r} .
$$

In obtaining the effective Hamiltonian (3.6), we have kept only those terms that are quadratic in the creation and annihilation operators, thus limiting its use to weakly interacting boson systems.

The effective quadratic Hamiltonian in Eq. (3.6) is more complicated than that of Bogolubov's Hamiltonian $[16,20]$ since $A$ and $B$ are full matrices, but it may be diagonalized by using the generalized canonical transformation in Eqs. (2.2) and (2.3). The matrix elements of $A \pm 2 B$, which appear in Eq. (2.9) (which determines the excitation spectrum as well as the transformation matrices), may be written using Eqs. (3.8)-(3.10) as a matrix representation of two different operators in the basis of the real eigenvectors of the trap potential,

$$
(A \pm 2 B)_{n m} \equiv\left\langle\phi_{n}\left|S_{ \pm}\right| \phi_{m}\right\rangle
$$

where

$$
S_{ \pm}=H_{0}+N g\left[(2 \pm 1) \phi_{0}^{2}-h_{00}\right]
$$

and

$$
H_{0}=\frac{P^{2}}{2 M}+U_{0}-\varepsilon_{0}^{0} .
$$

The EES is then given by the square root of the eigenvalues of either of the following equations [cf. Eq. (2.9)]:

$$
\begin{aligned}
& \left(S_{+} S_{-}\right) \Psi^{R}=E^{2} \Psi^{R}, \\
& \left(S_{+} S_{+}\right) \Psi^{L}=E^{2} \Psi^{L} .
\end{aligned}
$$

Since the product of $S_{-}$and $S_{+}$is no longer a symmetric operator, the eigenvalue problem for $S_{+} S_{-}$is a generalized eigenvalue problem and $\Psi^{L}$ and $\Psi^{R}$ are the left and right eigenvectors. Consequently, we see from Eq. (2.9), using Eqs. (3.11), (3.14), and (3.15), that

$$
\begin{aligned}
& (\alpha-\beta)_{l n}=\left\langle\phi_{l} \mid \Psi_{n}^{R}\right\rangle, \\
& (\alpha+\beta)_{l n}=\left\langle\Psi_{n}^{L} \mid \phi_{l}\right\rangle,
\end{aligned}
$$

where $\Psi_{n}^{R}$ and $\Psi_{n}^{L}$ are the right and left eigenfunctions corresponding to the eigenvalue $E_{n}^{2}$ and the $\phi_{l}$ are the trap eigenfunctions. Relation (2.6) is satisfied if the right and left eigenfunctions are normalized according to the condition

$$
\left\langle\Psi_{l}^{L} \mid \Psi_{n}^{R}\right\rangle=\delta_{l n}
$$

Thus

$$
\beta_{l n}=\frac{1}{2}\left[\left\langle\Psi_{n}^{L} \mid \phi_{l}\right\rangle-\left\langle\phi_{l} \mid \Psi_{n}^{R}\right\rangle\right]
$$

and

$$
\alpha_{l n}=\frac{1}{2}\left[\left\langle\Psi_{n}^{L} \mid \phi_{l}\right\rangle+\left\langle\phi_{l} \mid \Psi_{n}^{R}\right\rangle\right] .
$$

For a weakly interacting system, the second term on the right-hand side of Eq. (3.12), which stems from the interaction between bosons, may be treated as a perturbation. Thus

$$
S_{+} S_{-} \approx H_{0}^{2}+N g\left[H_{0} \phi_{0}^{2}+3 \phi_{0}^{2} H_{0}-2 h_{00} H_{0}\right]
$$

Then, solving the eigenvalue problem in Eqs. (3.14) and (3.15) gives, to first order,

$$
E_{n}=\left[\varepsilon_{n}^{2}+2 N g \varepsilon_{n}\left(2 h_{n n}-h_{00}\right)\right]^{1 / 2} .
$$

Equation (3.22) is similar to Bogolubov's result for a dilute hard-sphere boson gas in free space $[16,20]$, i.e.,

$$
E_{\vec{k}}=\left(\varepsilon_{\vec{k}}^{2}+2 n g \varepsilon_{\vec{k}}\right)^{1 / 2},
$$

where $\varepsilon_{\vec{k}}=k^{2} / 2 M$ is the energy of a free particle and $n$ is the density of particles. The main difference between Eqs. (3.22) and (3.23) is that the energies of the free particles in the latter are replaced by the energy levels of the trap in the former. Thus the trapped dilute boson gas will not follow a phonon law for the lowest part of the excitation spectrum. Also, Eq. (3.22) depends on the geometry of the trap through its dependence on the integrals $h_{n n}$. Of course, Eq. (3.22) is obtained using first-order perturbation theory and so it is accurate only as long as the second term under the square root in Eq. (3.22) is much smaller than the first term. Nevertheless, Eq. (3.22) serves to describe the behavior of the EES as a function of $N$, taking into account not only the sign of the scattering length [which enters $g$ according to Eq. (3.5)] but also the geometry of the trap.

\section{RESULTS FOR HARMONIC-OSCILLATOR POTENTIAL TRAPS}

For the case of harmonic-oscillator potential traps, the low-density EES in Eq. (3.22) may be calculated analytically using Eq. (3.4) for $g$ and Eq. (3.10) for $h_{n n}$. For a spherically symmetric harmonic-oscillator trap, one obtains

$$
\begin{aligned}
E_{n l}= & (2 n+l) \omega\left[1-\frac{4 a_{s c} N}{2 n+l}\left(\frac{M \omega}{2 \pi}\right)^{1 / 2}\right. \\
& \left.\times\left(1-\frac{(2 l+1) ! !}{(2 n+2 l+1) ! !} \frac{(l+3 / 2)_{2 n}}{n !} 2^{-n-l+1}\right)\right]^{1 / 2},
\end{aligned}
$$

where $n$ is the radial quantum number, $l$ is the orbital angular momentum quantum number, and $(n)_{k}$ denotes $n(n+1) \cdots(n+k-1)$. For a cylindrically symmetric harmonic-oscillator trap, with incommensurate axial $\left(\omega_{\|}\right)$ and radial $\left(\omega_{\perp}\right)$ frequencies, one obtains 


$$
\begin{aligned}
E_{n_{\perp} n_{\|}|m|}= & \varepsilon_{n_{\perp} n_{\|}|m|}\left[1-N a_{s c} \frac{4 \omega_{\perp}}{\varepsilon_{n_{\perp} n_{\|}|m|}}\left(\frac{M \omega_{\|}}{2 \pi}\right)^{1 / 2}\right. \\
& \left.\times\left(1-C_{2 n_{\perp}+|m|}^{n_{\perp}} C_{2 n_{\|} \|^{n}} 2^{-2 n_{\perp}-2 n_{\|}-|m|+1}\right)\right]^{1 / 2},
\end{aligned}
$$

where $\varepsilon_{n_{\perp} n_{\|}|m|}$, given by

$$
\varepsilon_{n_{\perp} n_{\|}|m|}=n_{\|} \omega_{\|}+\left(2 n_{\perp}+|m|\right) \omega_{\perp},
$$

designates the energy levels of the trap, the $C_{n}^{k} \equiv n ! / k !(n-k) !$ are the binomial coefficients, $n_{\perp}$ is the radial quantum number, $n_{\|}$is the axial quantum number, and $m$ is the axial orbital angular momentum quantum number. Finally, for an anisotropic harmonic-oscillator trap potential, with incommensurate frequencies, the EES is

$$
\begin{aligned}
E_{n_{1} n_{2} n_{3}}= & \varepsilon_{n_{1} n_{2} n_{3}}\left[1-\frac{4 N a_{s c}}{\varepsilon_{n_{1} n_{2} n_{3}}}\left(\frac{M \omega_{1} \omega_{2} \omega_{3}}{2 \pi}\right)^{1 / 2}\right. \\
& \left.\times\left(1-C_{2 n_{1}}^{n_{1}} C_{2 n_{2}}^{n_{2}} C_{2 n_{3}}^{n_{3}} 2^{-n_{1}-n_{2}-n_{3}+1}\right)\right]^{1 / 2},
\end{aligned}
$$

where the $\varepsilon_{n_{1} n_{2} n_{3}}$, given by

$$
\varepsilon_{n_{1} n_{2} n_{3}}=n_{1} \omega_{1}+n_{2} \omega_{2}+n_{3} \omega_{3},
$$

are the trap energy levels and $n_{1}, n_{2}$, and $n_{3}$ are the Cartesian quantum numbers. Note that in the derivation of Eq. (4.1) [Eqs. (4.2) and (4.4)] we have used the generating function for the Laguerre [Hermite] polynomials in order to compute the integrals $h_{n n}$ [cf. Eq. (3.10)].

\section{DISCUSSION}

In this paper we have presented in Eq. (3.22) an analytic expression for the EES for a trapped Bose-Einstein condensate that is valid in the low-density limit. Explicit formulas for the three possible kinds of harmonic-oscillator trap potentials are given in Eqs. (4.1), (4.2), and (4.4). Our analytic results for the low-density limit complement that of Stringari [10] for the high-density limit, whose result in this limit for the case of a spherically symmetric harmonic-oscillator trap is $[10]$

$$
E_{n l}^{S}=(2 n+l) \omega\left[\frac{2 n+1}{2 n+l}-\frac{n(2 n-1)}{(2 n+l)^{2}}\right]^{1 / 2} .
$$

Stringari's result in Eq. (5.1) is valid for $\eta \equiv N a_{s c}(M \omega)^{1 / 2} \gg 1$. Our result for the opposite limit $\eta \ll 1$ is given in Eq. (4.1). In contrast to Eq. (5.1), which shows no dependence on either the scattering length or on the number of particles, Eq. (4.1) shows that the $E_{10}$ level increases with $N$, while that for $E_{01}$ decreases with $N$ for $a_{s c}>0$. Also, our Eqs. (4.1), (4.2), and (4.4) show that the geometry of the trap has an important influence at low densities. As one example, note that in contrast to the case of a symmetric harmonic-oscillator trap [cf. Eq. (4.1)], Eq. (4.4)

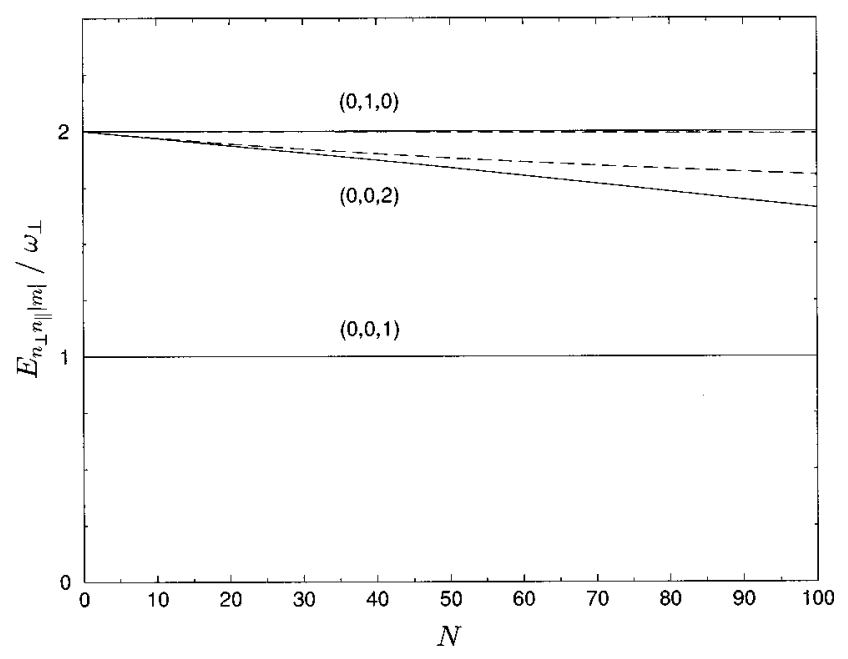

FIG. 1. Elementary excitation level energies $E_{n_{\perp} n_{\|}|m|}$ (in units of $\omega_{\perp}$ ) vs $N$, the number of trapped ${ }^{87} \mathrm{Rb}$ atoms, for the case of an axially symmetric, harmonic-oscillator trap having $\omega_{\|}={ }_{\sqrt{ }} 8 \omega_{\perp}$. The labels on the curves indicate the set of quantum numbers $\left(n_{\perp}, n_{\|},|m|\right)$. All results shown are for $a_{s c}=110$ a.u. and $\omega_{\perp}=74$ Hz. Solid curves, present perturbative analytic results using Eq. (4.2); dashed curves, nonperturbative numerical results of Edwards et al. $[11,21]$. Note that for the state $(0,0,1)$, our results and those of Edwards et al. cannot be distinguished.

for the anisotropic-oscillator trap configuration shows that all excited-state levels decrease with $N$ for $a_{s c}>0$.

In addition to their usefulness in predicting significant qualitative information on the influence of the particle density, scattering length, and trap geometry on the excitation energy-level spectrum for the limit of low trap densities, our Eqs. (4.1), (4.2), and (4.4) may also used as useful benchmarks for results of fully numerical calculations. In Figs. 1 and 2 we compare predictions of our Eq. (4.2) for the case of an axially symmetric harmonic-oscillator trap with results of the nonperturbative numerical calculations for ${ }^{87} \mathrm{Rb}$ of Edwards et al. [11] and of Esry [12], respectively,

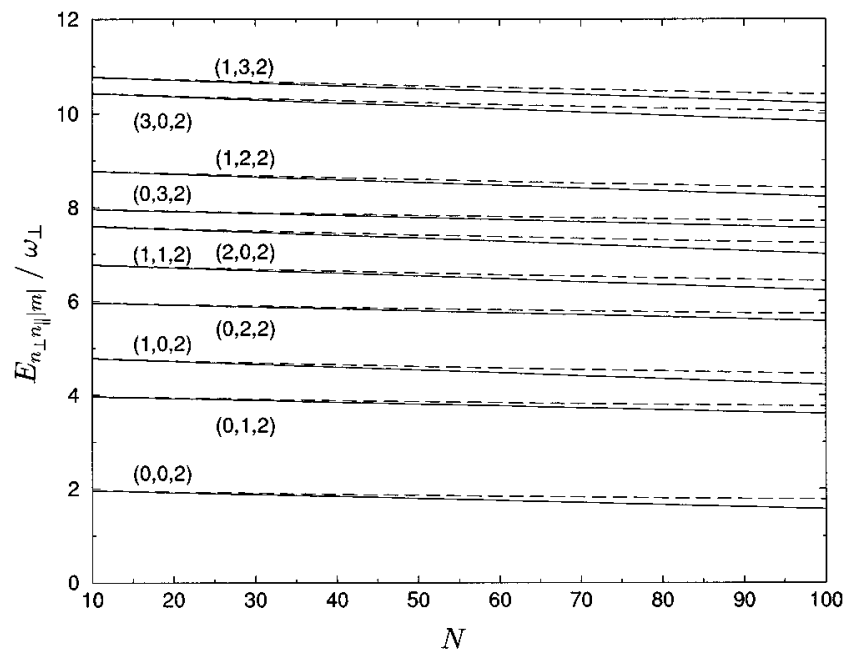

FIG. 2. Same as for Fig. 1, except that all results shown are for $a_{s c}=100$ a.u. and $\omega_{\perp}=133 \mathrm{~Hz}$ and here the dashed curves represent the nonperturbative numerical results of Esry [12,22]. 
which have been provided to us for the low-density region [21,22]. The results shown in Fig. 1 were obtained for $a_{s c}=110$ a.u. and $\omega_{\perp}=74 \mathrm{~Hz}$, while those in Fig. 2 are for $a_{s c}=100$ a.u. and $\omega_{\perp}=133 \mathrm{~Hz}$. In both cases $\omega_{\|}=\sqrt{8} \omega_{\perp}$. One sees from these figures that our perturbative analytic predictions and the predictions of the nonperturbative numerical calculations agree essentially exactly for $N \leqslant 30$. For higher numbers of trapped particles, our perturbative predictions are typically, but not always, lower than those of the nonperturbative numerical calculations, at least for the levels shown in these figures.

\section{ACKNOWLEDGMENTS}

We thank Mark Edwards and Brett Esry for providing us with the results of their calculations shown in Figs. 1 and 2 respectively. This work was supported in part by the U.S. Department of Energy, Division of Chemical Sciences, Office of Basic Energy Sciences, under Grant No. DE-FG0396ER14646.
[1] M. H. Anderson, J. R. Ensher, M. R. Matthews, C. E. Wieman, and E. A. Cornell, Science 269, 198 (1995).

[2] C. C. Bradley, C. A. Sackett, J. J. Tollett, and R. G. Hulet, Phys. Rev. Lett. 75, 1687 (1995).

[3] K. B. Davis, M.-O. Mewes, M. R. Andrews, N. J. van Druten, D. S. Durfee, D. M. Kum, and W. Ketterle, Phys. Rev. Lett. 75, 3969 (1995).

[4] S. Grossmann and M. Holthaus, Phys. Lett. A 208, 188 (1995).

[5] M. Lewenstein and L. You, Phys. Rev. A 53, 909 (1996).

[6] M. Edwards, R. J. Dodd, C. W. Clark, P. A. Ruprecht, and K. Burnett, Phys. Rev. A 53, R1950 (1996); P. A. Ruprecht, M. Edwards, K. Burnett, and C. W. Clark, ibid. 54, 4178 (1996).

[7] M. Holland and J. Cooper, Phys. Rev. A 53, R1954 (1996).

[8] F. Dalfovo and S. Stringari, Phys. Rev. A 53, 2477 (1996).

[9] D. S. Jin, J. R. Ensher, M. R. Matthews, C. E. Wieman, and E. A. Cornell, Phys. Rev. Lett. 77, 420 (1996).

[10] S. Stringari, Phys. Rev. Lett. 77, 2360 (1996).

[11] M. Edwards, P. A. Ruprecht, K. Burnett, R. J. Dodd, and C.
W. Clark, Phys. Rev. Lett. 77, 1671 (1996).

[12] B. D. Esry, Phys. Rev. A 55, 1147 (1997).

[13] S. V. Tyablikov, Methods in the Quantum Theory of Magnetism (Plenum, New York, 1967), pp. 105-113.

[14] M. Alexanian and R. A. Brito, Phys. Rev. B 17, 3547 (1978).

[15] J.-P. Blaizot and G. Ripka, Quantum Theory of Finite Systems (MIT Press, Cambridge, MA, 1986), pp. 58-68.

[16] N. Bogolubov, J. Phys. (Moscow) 11, 23 (1947).

[17] M. D. Girardeau, J. Math. Phys. (N.Y.) 16, 1901 (1975).

[18] T. D. Lee, K. Huang, and C. N. Yang, Phys. Rev. 106, 1135 (1957).

[19] N. G. van Kampen, Phys. Rev. 135, A362 (1964).

[20] A. L. Fetter and J. D. Walecka, Quantum Theory of ManyParticle Systems (McGraw-Hill, New York, 1971), pp. 314319.

[21] M. Edwards (private communication).

[22] B. Esry (private communication). 